\title{
Associations between dietary intake and volumetric bone mineral density in South Asian and Caucasian women: preliminary analysis of the D2-D3 Study
}

\author{
L. Tripkovic, L. Wilson, K. Hart and S. Lanham-New \\ Department of Nutritional Sciences, Faculty of Health and Medical Sciences, University of Surrey, Guildford GU2 7XH, UK
}

The evidence base confirming the necessity of sufficient vitamin D (in addition to other nutrients such as calcium, phosphate and magnesium) in order to maintain skeletal health is well established. However, within the UK, previous data has shown that up to $\approx 87 \%$ of the population could have sub-optimal vitamin D status during the winter and early-spring months ${ }^{(1)}$. In the diet, few foods naturally contain vitamin $\mathrm{D}^{(2)}$. Thus the majority of the UK population rely on sunshine as their main source of vitamin $\mathrm{D}$ which is problematic since during the months of Oct-Mar in the UK, the angle of the sun results in UVB rays that are not at optimum length (290-320 nm) to penetrate the skin and allow vitamin D production.

The primary aim of the D2-D3 Study (a double blind, food fortification RCT) is to compare the efficacy of $15 \mu \mathrm{g} / \mathrm{d}$ Vitamin D2 vs. $15 \mu \mathrm{g} / \mathrm{d}$ Vitamin D3 in raising 25(OH)D levels in both Caucasian and South Asian women during the winter and early spring months. With the sample collection recently completed, preliminary analysis of the baseline data is now possible with the aim to assess for potential associations between skeletal health and dietary intake for both South Asian and Caucasian women.

A cohort of 335 healthy women (Caucasian $n$ 245, South Asian $n$ 90) with a mean age of $43.6 \pm 12.3$ years and BMI of $24.1 \pm 3.8 \mathrm{~kg} /$ $\mathrm{m}^{2}$ were recruited to the D2-D3 Study over the course of two consecutive winters (Oct 2011-Jan 2012 and Oct 2012-Jan 2013). At the baseline study visit, anthropometrics and a peripheral quantitative computed tomography (pQCT) scan of the radius were completed. The participants were also requested to complete a detailed record of their dietary intake on four consecutive days.

Comparing ethnicity groups for body composition, the Caucasian (CA) women were significantly taller $(p<0.001)$ than the South Asian (SA) women. The CA women also had a significantly lower BMI, waist circumference, waist:hip ratio and body fat percentage $(p<0.01)$ than the SA women. There was no significant difference in weight between the two population groups $(\mathrm{CA}$ : $64.7 \pm 10.1 \mathrm{~kg}$, SA: $64.3 \pm 10.9 \mathrm{~kg}$ ). Dietary analysis indicated that for an average daily intake, the CA and SA women consumed similar amounts of energy (CA: $8045.9 \pm 2045.7 \mathrm{~kJ} /$ day, SA: $7675.3 \pm 1816.6 \mathrm{~kJ} /$ day). However the CA women consumed significantly greater quantities of saturated and trans fat, fibre, sodium, potassium, calcium, magnesium, phosphate, iodine and alcohol $(p<0.05)$ than SA women. The SA women consumed significantly greater quantities of vitamin $\mathrm{K} 1$ than $\mathrm{CA}$ women $(P<0.04)$.

Analysis of the pQCT data indicated that the CA women had significantly greater bone mass $(P<0.04)$ and area $(P<0.001)$ at the distal radius ( $4 \%$ site) compared to the SA women. Yet at the same site, the SA women had significantly greater volumetric bone mineral density (vBMD) than the CA women $(P<0.009)$.

When focusing on vitamin $\mathrm{D}$, the comparison of tertiles of vitamin D intake and the association with vBMD found no clear relationship for either group. Nor were there any significant correlations between vitamin D intake and vBMD. However for CA women, significant negative correlations were found between alcohol intake and bone mass, vBMD and trabecular density at the $4 \%$ site and cortical density at the $66 \%$ site $(P<0.04)$.

Further analysis of these diet/bone health associations are currently underway, particularly with respect to the higher vBMD detected in SA women despite a lower intake of the nutrients required for optimum bone health when compared with the CA women. Consideration of the impact of age, menopausal status and body composition upon bone health outcomes for both SA and CA women is also a priority.

This work is funded by the BBSRC DRINC Programme (Grant No. BB/I006192/1).

1. Hyponnen \& Power (2007) Am J Clin Nutr 85, 860-68.

2. Holick (2007) New Eng $J$ Med 357, 266-81. 